\title{
Optimal Management of the Spare Parts Stock at Their Regular Distribution
}

\author{
Svetozar Madzhov \\ Forest Research Institute, Bulgarian Academy of Sciences, Sofia 1756, Bulgaria
}

\begin{abstract}
The article presents a model for optimal management of the SP (Spare Parts) stock at their regular distribution. The present research discussion is a multi-nomenclature model with a fixed limit of the stock's value. A study was carried out on the optimal value of storage cost for a unit of stock at a unit of time $\left(h_{j}\right)$, optimal value of the demand intensity $\left(\lambda_{j}\right)$ and the optimal price of a stock unit $\left(c_{j}\right)$, subject to the restriction for the upper limit $K$ of the total value of stocks.
\end{abstract}

Key words: SP, optimum management, stock, forestry.

\section{Introduction}

The question of the optimal stock management has been discussed in one way or another in many publications [1-6]. Most often, mono nomenclature determinant models are discussed. They are of interest, because they enable us to study the ideology of research methods used in more sophisticated systems. Practically, a large number of different types of stocks are stored in the warehouses, which imposes that different kinds of nomenclature models for stock management of SP (Spare Parts) are developed. Another important feature of the SP management system in the practice is the presence of restrictions, more total number of deliveries during the year, the maximal storage space, maximal volume of each separate delivery, minimal quantity of the ordered production, etc.

The companies in the real economy usually do not dispose of unlimited financial resource, to be invested in stock, but just the opposite-they dispose of a limited working capital, by which they maintain the stock. The present review studies a multi-nomenclature model with a preliminary defined limitation of the stocks' value. The conducted research of the optimum

Corresponding author: Svetozar Madzhov, Assistant Professor, Ph.D., research field: Forest research. cost values for a unit storage for a unit of time $\left(h_{j}\right)$, the optimum value of the demand intensity $\left(\lambda_{j}\right)$ and the optimum price for a stock unit $\left(c_{j}\right)$, upon keeping the upper limit $(K)$ of the total stocks' value is presented in Figs. 1-3 respectively. The output data are collected in the led in the town of Elin Pelin research for $\lambda_{j}$, and the other data are borrowed by Ref. [7], with updated prices.

\section{Materials and Methods}

Let us assume that $n$ kinds of stocks are stored in the warehouse and there is always an upper limit $K$ of their total value. If we assume and designate the deliveries' $j$ kind volume by $Q_{j}$, then the limitation may be written down by the inequality:

$$
c_{1} Q_{1}+c_{2} Q_{2}+\ldots \ldots+c_{n} Q_{n} \leq \alpha K
$$

Whereas $\alpha$ is the revaluation factor due to the irregularity of some deliveries' types, $\alpha \in(0,1)$, $\mathrm{c}_{j}$ - the price of a stock. Let us assume that $\lambda_{j}$ is the intensity of demand of products of the kind $j$ (the demand is determinant and with a regular intensity within the time), $A_{j}$ is the constant content of deliveries' costs, $\mathrm{h}_{\mathrm{j}}$ - the storage costs for a stock unit for a time unit. The model does not allow unsatisfied demand of neither of the stocks' types. Therefore the average costs for a time unit for stocks of the type $j$ 
shall be illustrated by the equation of the Wilson model [2-5]:

$$
\frac{\lambda_{j}}{Q_{j}} A_{j}+h_{j} c_{j} \frac{Q_{j}}{2}+\lambda_{j} c_{j},
$$

And the total costs of all stocks shall be expressed by the Eq. (2):

$$
R=\sum_{j=1}^{n}\left[\frac{\lambda_{j}}{Q_{j}} A_{j}+h_{j} c_{j} \frac{Q_{j}}{2}\right]+\sum_{j=1}^{n} \lambda_{j} c_{j}
$$

It is necessary to find such numbers $Q_{1}^{*} \geq 0, \ldots, Q_{n}^{*} \geq 0$, which substituted in Eq. (2) to give the maximal value of the total costs $R$. The latest sum in Eq. (2) does not depend on the manageable parameters $Q_{1}, \ldots, Q_{n}$ and will not influence the solution of the task. The sum of the positive addend will be minimal, if each of the addends reaches its maximal value. But for the addend $j$ this value will be reached below in Ref. [3]:

$$
Q_{j}=\sqrt{2 \lambda_{j} A_{j} / h_{j}}, j=1,2, \ldots, n
$$

If these numbers $Q_{j}$ satisfy the limitation (1), then they are the optimal ones.

In this case the limitation (1) is insignificant, i.e. the capital investments are enough and their increase will not lead to decrease of the total costs. If on the other hand, $Q_{j}$ do not satisfy Eq. (1), the limitation of the stock value is significant and the optimal $Q_{j}^{*}$ are others.

In the last case, for getting the optimal $Q_{j}^{*}$ the Lagrange method of the undefined multiplier shall be applied. Authors are forming the Lagrange function:

$$
\mathfrak{I}=\sum_{j=1}^{n}\left[\frac{\lambda_{j}}{Q_{j}} A_{j}+h_{j} \frac{Q_{j}}{2}\right]+\omega\left(\sum_{j=1}^{n} c_{j} Q_{j}-\alpha K\right)
$$

where $\omega$ is Lagrange's undefined multiplier.

The set of numbers $Q_{j}^{*}, j=1, \ldots, n$, at which the function $R$ has an absolute minimum and satisfies the limitation Eq. (1), is the solution of the equation system.

$$
\left\{\begin{array}{c}
\frac{\partial \mathfrak{I}}{\partial Q_{j}}=-\frac{\lambda_{j}}{Q_{j}^{2}} A_{j}+\frac{1}{2} h_{j}+\omega c_{j}=0, \quad j=1, \ldots, n \\
\frac{\partial \mathfrak{I}}{\partial Q_{j}}=\sum_{j=1}^{n} c_{j} Q_{j}-\alpha K=0 .
\end{array}\right.
$$

System (3) has one single solution:

$$
Q_{j}(\omega)=\sqrt{\frac{2 \lambda_{j} A_{j}}{h_{j}+2 \omega c_{j}}} ; \quad j=1,2, \ldots, n .
$$

and to fulfill also the last condition (3), it is necessary to be selected in such a way that:

$$
K(\omega)=\sum_{j=1}^{n} c_{j} \sqrt{\frac{2 \lambda_{j} A_{j}}{h_{j}+2 \omega c_{j}}}=\alpha K
$$

which is new and allows optimizing the SP stock management.

\section{Results and Discussion}

The conducted research of the optimum cost values for a unit storage for a unit of time $\left(h_{j}\right)$, the optimum value of the demand intensity $\left(\lambda_{j}\right)$ and the optimum price for a stock unit $\left(c_{j}\right)$, upon keeping the upper limit $(K)$ of the total stocks' value is presented in Figs. 1-3 respectively. The allowed values of the presented parameters belong to the multiplicity $A$, and the not allowed - of the multiplicity $B$. The optimum value is located on the cross point of the graphic of the functions of the upper limit $(K)$ of the total value of the storages and the constant component of delivery costs $(A)$ (Fig. 1), the value of the demand intensity $\left(\lambda_{j}\right)$ (Fig. 2.) and the price of a stock unit ( $\left.c_{j}\right)$ (Fig. 3).

As the left hand side of eq. (4) is a monotonously decreasing function of $\omega$ and $K(0)>\alpha K$, upon the known $\alpha$ and $K$, Eq. (4) has one single solution $\omega^{*}$ in regards to the parameter $\omega$. The management strategy upon the presence of the limitation (1) is defined by the quantities of $Q_{j}^{*}=Q_{j}(\omega)$, calculated by equality (3). The numerical algorithm for the determination of $\omega^{*}$ turns out under the following algorithm: 


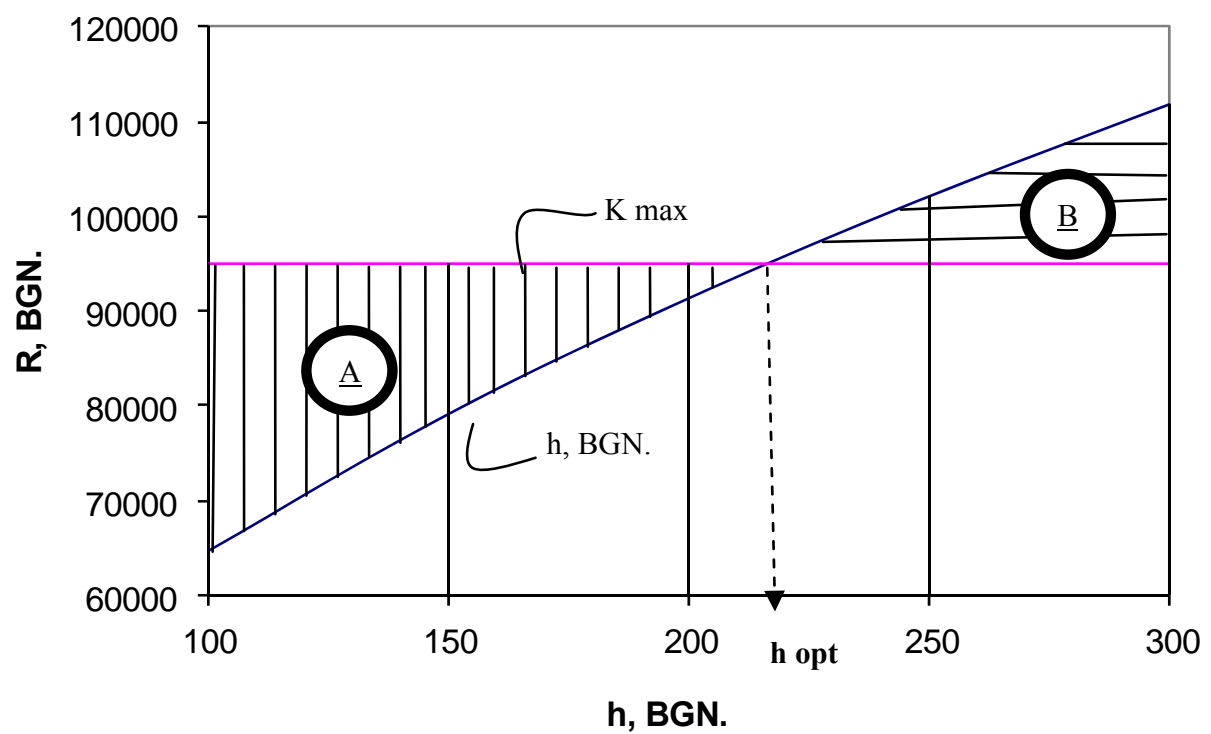

Fig. 1 The optimum costs value for storage of a stock unit for a unit of time $\left(h_{j}\right)$, upon keeping the upper limit $(K)$ of the total stocks' value; A-the multiplicity of allowable storage values for a storage of a stock unit for a unit of time $\left(h_{j}\right)$; B-the multiplicity of unacceptable values of storage of a stock unit for a unit of time $\left(h_{j}\right)$.

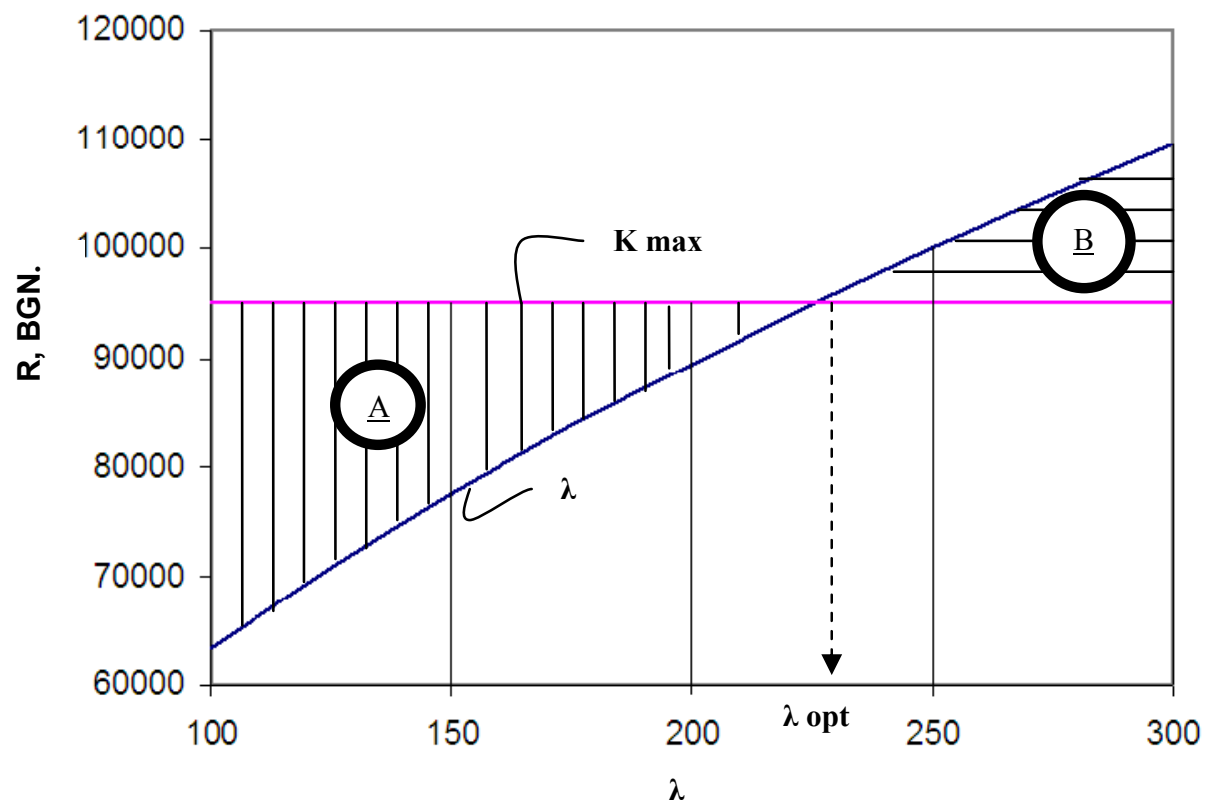

Fig. 2 The optimum value of the demand intensity $\left(\lambda_{j}\right)$, upon keeping the upper limit $(K)$ of the total stocks' value; A-multiplicity of the allowed values of demand intensity $\left(\lambda_{j}\right)$; B-the multiplicity of unacceptable values of demand intensity $\left(\lambda_{j}\right)$. 


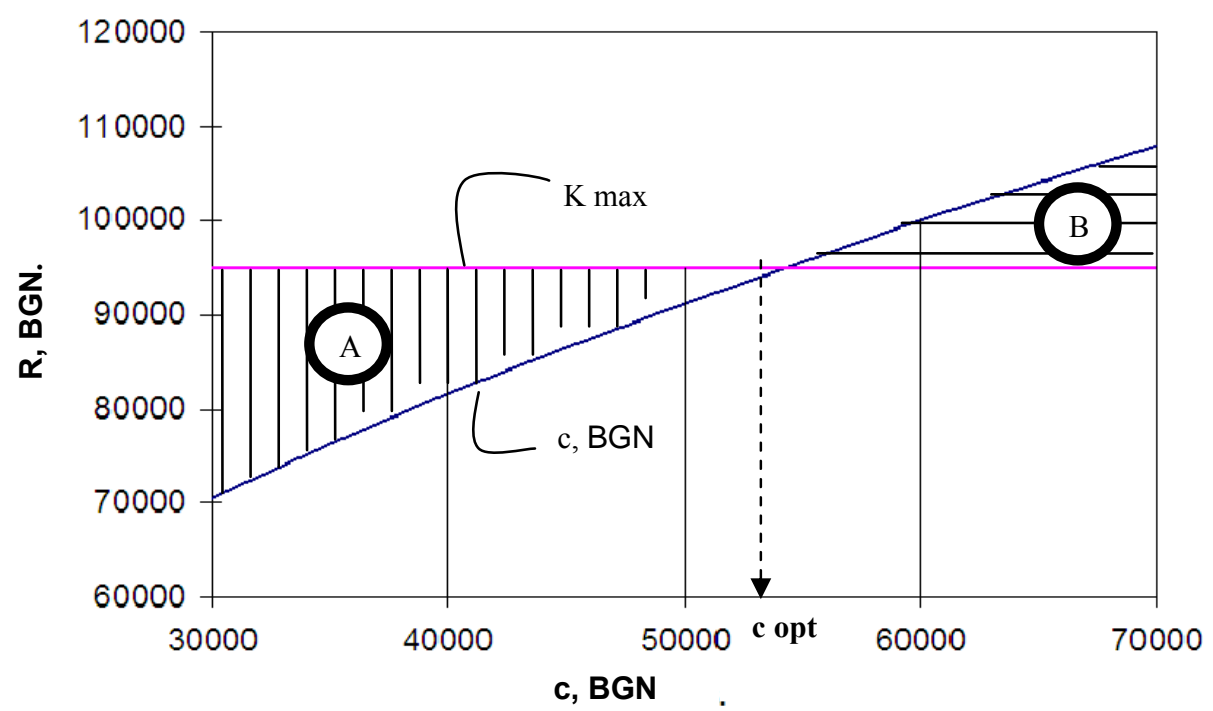

Fig. 3 The optimum value of price for a unit of stock $\left(c_{j}\right)$, upon keeping the upper limit $(K)$ of the total stocks' value; A-multiplicity of the allowed values of the price for a unit of stock $\left(c_{j}\right)$, B-the multiplicity of unacceptable values of the price for a unit of stock $\left(c_{j}\right)$.

Authors choose one random value $\omega>0$ and calculate $K\left(\omega_{1}\right)$.

We compare $K\left(\omega_{1}\right)$ with $\alpha K$

(1) If $K\left(\omega_{1}\right)=\alpha K$, then $\omega^{*}=\omega_{1}$ and the searched $Q_{j}^{*}$ are $Q_{j}^{*}=Q_{j}(\omega)$, and the calculations end.

(2) If $K\left(\omega_{1}\right)>\alpha K$, authors choose such a value $\omega_{2}>$ $\omega_{1}$ to calculate $K\left(\omega_{2}\right)$ and proceed to a comparison of $K\left(\omega_{2}\right)$ with $\alpha K$, i.e. to the preceding point of the newly chosen value of $\omega$.

(3) If $K\left(\omega_{1}\right)<\alpha K$, authors choose $\omega_{2}<\omega_{1}$, to calculate $K\left(\omega_{2}\right)$ and proceed to point 2 for the new $\omega=\omega_{2}$.

The optimization procedure may end, when the current solution $\omega_{n}$ is considered satisfactory, i.e. when $\left|K\left(\omega_{n}\right)-\alpha K\right|<\varepsilon(\varepsilon>$ is an acceptably small value). In order to use this model in the practice we should know the fluctuation of its following parameters: upper limit of the stock value $(K \mathrm{w})$, stock deliveries' volume by the j-kind $\left(Q_{j}\right)$ and the total costs of all stocks $(R)$.

The research object is the fluctuation of the model parameters, in relation to the management factors: demand intensity of products of the $j$-kind $\left(\lambda_{j}\right)$, constant costs component $\left(A_{j}\right)$, storage costs $\left(h_{j}\right)$, stock unit's price $\left(c_{j}\right)$ and the indefinite multiplier of Lagrange $\left(w_{j}\right)$.

Figs. 4-6 show the fluctuation of the total costs of all stocks $(R)$ depending on the fluctuation of deliveries' volume of sock of $j$-kind $\left(Q_{j}\right)$ and the intensity of demand of products of $j$-kind $\left(\lambda_{j}\right)$. The other parameters, namely the constant content of costs $\left(A_{j}\right)=20 \mathrm{BGN}$, storage costs $\left(h_{j}\right)=20 \mathrm{BGN}$ and the price of a unit of stock $\left(c_{j}\right)=30 \mathrm{BGN}$ are assumed constant [8]. It is obtained by regression analysis and described in Fig. 4 that with the increase of the deliveries' volume of the stock $j-\left(Q_{j}\right)$ the costs $R_{j}$ are increased linearly, and the curves of $R=\mathrm{f}\{Q\}$ are shifted in the direction increase of total costs of all stocks $(R)$. There is an increase in $R$ with an increase in the intensity of demand of products of $j$-kind $\left(\lambda_{j}\right)$. Fig. 5 shows that their change is also linear, but the increase of the volume of deliveries of stock of $j$-kind $\left(\mathrm{Q}_{j}\right)$ influences more significantly on $R=\mathrm{f}\{\lambda\}$.

A greater interest represents the fluctuation of the total costs $(R)$, when the two parameters change: stock deliveries, volume of $j$-type $\left(\mathrm{Q}_{j}\right)$ and the intensity of the product demand of $j$-type $\left(\lambda_{j}\right)$. As a rule, the total 
costs for all stocks $(R)$ increase linearly (Fig. 6), but with the increase in the stock volume of $j$-type $\left(\mathrm{Q}_{j}\right)$ of the costs $\mathrm{R}_{J}$-increase faster than in the case of increase of the intensity of demand of $j$-type products $\left(\lambda_{j}\right)$. Accordingly, the total costs for all stocks $(R)$ increase slower with the increase of the intensity of demand of $j$-type products $\left(\lambda_{j}\right)$, the total costs for all stocks $(R)$ have a maximal value of the volume of the stock delivery of the $j$-type $\left(Q_{j}\right)$ and the intensity of demand of products of the $j$-type $\left(\lambda_{j}\right)$, than in the opposite case, consequently the volume of supplies of $j$-type stock $\left(Q_{j}\right)$ influences more over the total costs of all stocks $(R)$, than the demand intensity of products of the $j$-type $\left(\lambda_{j}\right)$.

The next study describes the change of the total costs for all stocks $\left(R_{j}\right)$ in relation to the constant component of costs $\left(A_{j}\right)$ and the price of a unit stock $\left(c_{j}\right)$. The fluctuation of the deliveries' volume of stock of $j$-type $\left(Q_{j}\right)$, storage costs $\left(h_{j}\right)$ and product of the $j$-type demand intensity $\left(\lambda_{j}\right)$ are considered constants. Their values are: $Q_{j}=400$ items, $h_{j}=25 \mathrm{BGN}$ and $\lambda_{j}$ $=30$ items $[[8,9]$.

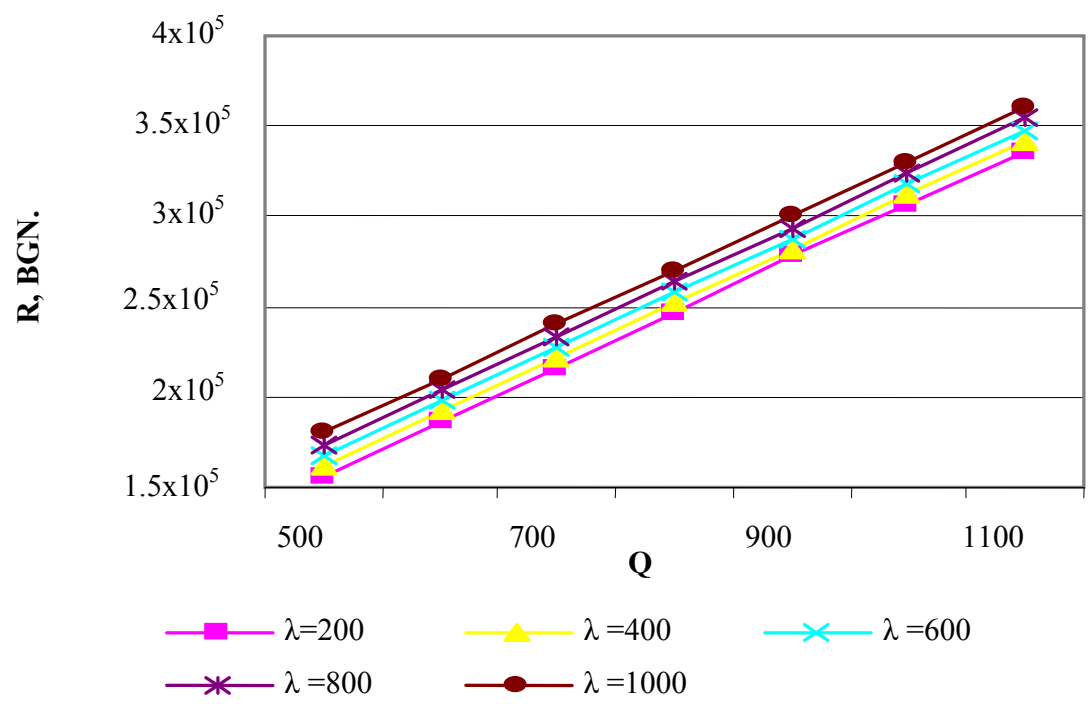

Fig. 4 Fluctuation of the total stock costs $\left(R_{j}\right)$ in relation to the deliveries' volume of the stock of the type $j\left(Q_{j}\right)$ at a constant component of costs $\left(A_{j}\right)=20$, storage costs $\left(h_{j}\right)=20$ and price of a stock unit $\left(c_{j}\right)=30$.

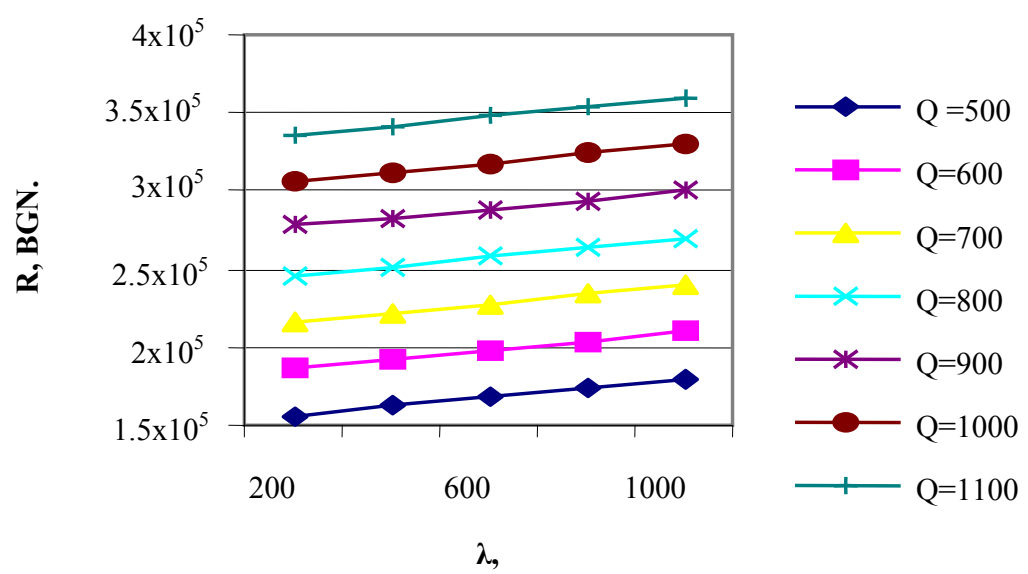

Fig. 5 Fluctuation of the total stock costs $\left(R_{j}\right)$ in relation to intensity of products of the type $j\left(\lambda_{j}\right)$ at a constant cost component $\left(A_{j}\right)=20$, storage costs $\left(h_{j}\right)=20$ and price of a stock unit $\left(c_{j}\right)=30$. 


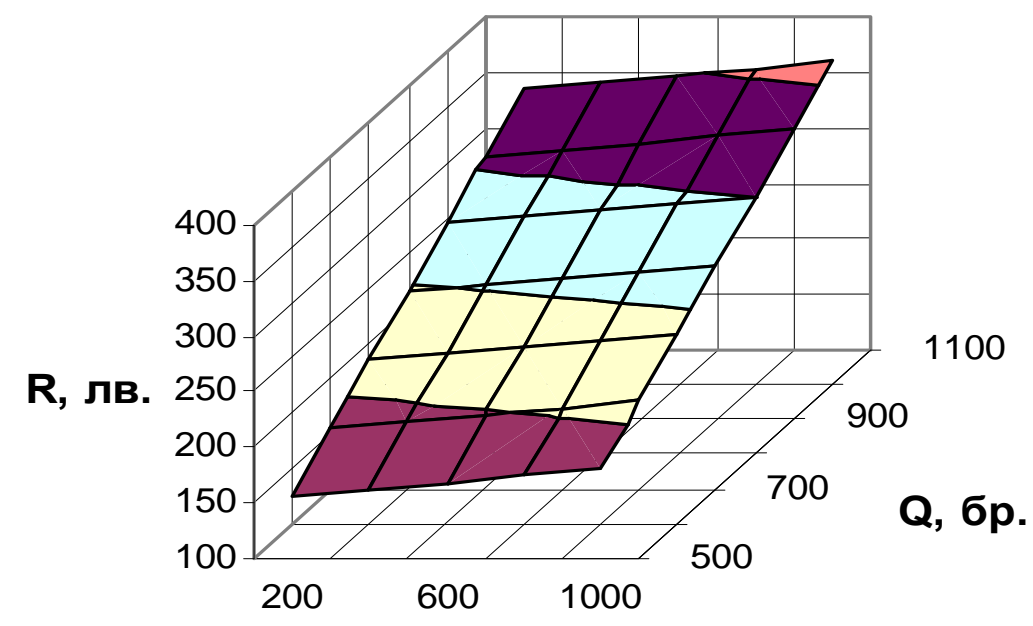

$\lambda, 6 p$.

Fig. 6 Fluctuation of the total stock costs $\left(R_{j}\right)$ in relation to the fluctuation of the volume of stock deliveries of the type $j\left(Q_{j}\right)$ and the demand intensity of products of the type $j\left(\lambda_{j}\right)$ at a constant $\left(A_{j}\right)=20$, storage costs $\left(h_{j}\right)=20$ and price of a stock unit $\left(c_{j}\right)=30$.

The fluctuation of the total costs of all stocks $(R)$ depending on the price for a unit of stock $\left(c_{j}\right)$ has been studied. The analysis of results (Fig. 4) indicates that the stock unit price increase $\left(c_{j}\right)$ leads to a linear increase of costs $R_{j}$, and the fluctuation of the constant component of costs do not influence on $R=\mathrm{f}(Q)$ (Fig. 5).

The study, conducted at a simultaneous fluctuation of the constant component of costs $\left(A_{j}\right)$ and the price of a stock unit $\left(c_{j}\right)$ (Fig. 6) over the total costs of al stocks $\left(R_{j}\right)$ shows that with the increase of price of a stock unit $\left(c_{j}\right)$ the costs $R_{j}$ increase quickly, but with the increase of the constant component of costs $\left(A_{j}\right)$ the costs $R$ increase insignificantly. The total fluctuation of the total costs of all stocks $(R)$ is shown as a surface, which is inclined at a certain angle to a level of three-dimensional width A - c.

\section{Conclusions}

A mathematical model for SP stock optimization at their regular distribution for maintenance of machine efficiency has been elaborated.

The influence of the main parameters of the model over the fluctuation of the total costs has been studied.

\section{References}

[1] Barlow, R., Proshan, F. 1984. Statistical Theory of Reliability and Reliability Tests.-M Holt,Rinehart \& Winston of Canada Ltd,. p. 328.

[2] Dimitrov, B. 1984. Scientific Inventory Management-Sofia. (in Bulgarian)

[3] Konstantin, G., Aleksandrov, K., et al. 2002. Survey of Operations-Sofia. (in Bulgarian)

[4] Ryzhikov, Y. 1969. Inventory Management-Moskow. (in Russian)

[5] Sakovich, V. 1986. Models of Inventory Management. Minsk. (in Russian)

[6] Spiridonov, G., and Tasev, G. 1984. Collection of Methodical and Normative Materials on the Maintenance of the Agricultural Machinery. Rousse. (in Bulgarian)

[7] Spiridonov, G., and Tasev, G. 1986. Justification of the Parameters of the System for Distribution of Spare Parts between the Units of ROSB. Rousse, Report on the subject No. 8530/1 (not published). (in Bulgarian)

[8] Tasev, G., and Antonov, B. 1988. Basic Issues in Regulating and Managing Stocks of Spare Parts, FTS, 7, pp. 46-53. Rousse (in Bulgarian)

[9] Spyridonov, G., and Tasev, G. 1981. Some Theoretical and Applied Aspects of the Repair and Maintenance of Agricultural Machinery. Rousse. (in Bulgarian) 